\title{
When do Projections Commute?
}

\author{
W. Rehder \\ Technische Universität Berlin
}

Z. Naturforsch. 35a, 437-441 (1980); received November 17, 1979

\begin{abstract}
Necessary and sufficient conditions for commutativity of two projections in Hilbert space are given through properties of so-called conditional connectives which are derived from the conditional probability operator PQP. This approach unifies most of the known proofs, provides a few new criteria, and permits certain suggestive interpretations for compound properties of quantum-mechanical systems.
\end{abstract}

\section{Introduction}

Commutativity of two projections $P$ and $Q$ in a complex Hilbert space $H$ plays an important rôle in the mathematical formulation and physical interpretation of quantum-mechanical systems. $P Q=Q P$ is interpreted as "commensurability" of the properties represented by $P$ and $Q$. This means: On a quantum-mechanical system in a given state, measurement of $P$ and $Q$ can be made simultaneously or, a measurement of first $P$ and then $Q$ affects any state $\varphi$ in the same way as does a measurement first of $Q$ and then of $P$ :

$$
\langle\varphi, Q P \varphi\rangle=\langle\varphi, P Q \varphi\rangle \text {. }
$$

Mathematically speaking, this identity is equivalent to the fact that $P Q$ is a projection onto the meet of $P$ and $Q$, which in turn means physically that $P Q(=Q P)$ is again a "compound" property of the system.

On the other hand, the meet (which by abuse of language we write $P \wedge Q$ ) of $P$ and $Q$ is uniquely defined even for non-commuting projections. In this case, $P Q$ is not a projection and a fortiori $P Q \neq Q P . P Q$ is not even an observable (hermitian operator) in $H$ and hence is not interpreted in quantum mechanics. The interpretation of $P \wedge Q$, however, has been controversial (see Jammer's book [4], p. 353-361).

The purpose of this paper is to present a somewhat unified approach to commutativity proofs for two projections in Hilbert space. We shall derive necessary and sufficient conditions for commutativity from properties of the so-called "con-

Reprint requests to Dr. Wulf Rehder, Fachbereich Mathematik HAD 3, Technische Universität Berlin, Straße des 17. Juni 135, 1000 Berlin 12. ditional probability operator" $P Q P$ (cf. Bub's discussion in [3], and the relevant literature quoted there). This observable leads to the introduction of derived connectives $P \sqcap Q, P \sqcup Q$, and the material quasi-implication $P \rightarrow Q$. These connectives allow a reasonable physical interpretation for the meet $P \wedge Q$ even for non-commuting projections. Most of the following material can be proved in the more general setting of quasimodular orthocomplemented lattices (cf. [9] and [10]). These criteria are rephrasings of known results in terms of the new connectives; only (3.17) below appears to be new.

\section{Conditional Connectives}

Let $P$ and $Q$ be projections in a complex Hilbert space $H$. Because of the one-to-one correspondence between projections and their ranges, we denote the range of $P$ by $P$ as well, so that

$$
P x=x \quad \text { and } \quad x \in P
$$

have the same meaning.

Let $E_{0}(P Q P)$ denote the null-space of $P Q P$ :

$$
E_{0}(P Q P)=\{x \in H \mid P Q P x=0\} .
$$

\subsection{Definition}

The "conditional" connective $P \sqcap Q$ is (the projection onto) the orthocomplement of $E_{0}(P Q P)$ :

$$
P \sqcap Q=E_{0}^{\perp}(P Q P),
$$

read " $P$ and then $Q$ ".

In other words, $P \sqcap Q$ is the projection onto the range of $P Q P$.

It follows from $\langle x, P Q P x\rangle=\|Q P x\|^{2}$ that $P Q P x=0$ if $Q P x=0$. Now the following representation of $E_{0}(P Q P)=E_{0}(Q P)$ ensues:

0340-4811 / $80 / 0400-0437 \$ 01.00 / 0$. - Please order a reprint rather than making your own copy. 


\subsection{Theorem}

For all projections $P, Q$ in $H$

$$
E_{0}(P Q P)=P^{\perp} \vee\left(P \wedge Q^{\perp}\right),
$$

where $\vee$ may be replaced by + .

Proof. $Q P x=0$ is equivalent to $P x \in Q$, and this is certainly fulfilled for all $x$ in the subspace on the right-hand side.

Conversely, if $x$ is such that $P x \in Q$, then we see from $x=(I-P) x+P x$ and $I-P=P^{\perp}$ that $x$ belongs also to the right-hand side of the equality above.

\subsection{Corollary}

$$
P \sqcap Q=P \wedge\left(P^{\perp} \vee Q\right) .
$$

2.3 justifies our reading of $P \sqcap Q$ as " $P$ and then $Q$ "; for, if we interpret the right-hand side of (2.3) via "classical" connectives, we see that $P \sqcap Q$ is true iff $P$ is true and it is true that $Q$ follows "materially" from $P$. It is now clear how to define $P \sqcup Q$ : " $P$ or then $Q$ ", and a material "quasiimplication" or "conditional implication" $P \rightarrow Q$ :

\subsection{Definitions}

For projections $P, Q$ in $H$ put

$$
\begin{aligned}
P \sqcup Q & :=\left(P^{\perp} \sqcap Q^{\perp}\right)^{\perp} \\
& =E_{0}\left(P^{\perp} Q^{\perp} P^{\perp}\right) \\
& =\left\{x \mid Q^{\perp} P^{\perp} x=0\right\} \\
& =P \vee\left(P^{\perp} \wedge Q\right) ; \\
P \rightarrow Q & :=P^{\perp} \sqcup Q \\
& =E_{0}\left(P Q^{\perp} P\right)=\left\{x \mid Q^{\perp} P x=0\right\} \\
& =P^{\perp} \vee(P \wedge Q) .
\end{aligned}
$$

$P \rightarrow Q$ can be read as follows: " $P \rightarrow Q$ is true iff either $P^{\perp}$ is true or the occurrence of the yesoutcome of $P$ leaves the system in a state which makes true $Q . "$ (cf. [2], p. 378).

\subsection{Corollary}

For all projections $P, Q$ in $H$ (commuting or not), we have

(1) $P \wedge Q \leqq P \sqcap Q$,

(2) $P \vee Q \geqq P \sqcup Q$,

(3) $P \wedge Q=P \sqcap\left(P^{\perp} \sqcup Q\right)=Q \sqcap\left(Q^{\perp} \sqcup P\right)$,

(4) $P \vee Q=P \sqcup\left(P^{\perp} \sqcap Q\right)=Q \sqcup\left(Q^{\perp} \sqcap P\right)$, note that $P \sqcup\left(P^{\perp} \sqcap Q\right)=P+\left(P^{\perp} \sqcap Q\right)$.
Proof. (1) and (2) are clear from (2.3) and (2.4). We prove (4): Since

$$
P \leqq P \vee Q, \quad P \vee Q-P=(P \vee Q) \wedge P^{\perp} .
$$

(3) follows from (4).

$P \wedge Q$ and $P \vee Q$ can also be expressed in terms of the spectral measure of the observable $P Q P$ :

\subsection{Theorem}

For all projections $P, Q$ in $H$

$$
P \wedge Q=E_{1}(P Q P)=E_{1}(Q P Q),
$$

where $E_{1}$ is the respective projection onto the eigenspace with the eigenvalue 1 .

Proof. $x \in E_{1}(P Q P)$ iff $P Q P x=x$. From $\|x\|^{2}=$ $\langle P Q P x, x\rangle=\|Q P x\|^{2} \leqq\|P x\|^{2} \leqq\|x\|^{2}$ we see that $P x=x$, i.e. $x \in P$, and also $Q P x=x$, and together with $P x=x$, that $Q x=x$. The converse is evident.

2.5 (3) allows a suggestive reading of the meet $P \wedge Q$, whether $P$ and $Q$ commute or not:

$P \wedge Q$ iff $P$ and then $P$ quasi-implies $Q$, which is the same as $Q$ and then $Q$ quasi-implies $P$.

\section{Commutativity of Projections}

$$
\begin{gathered}
\text { Abbreviate } P \sim Q \text { for } P Q=Q P . \text { Obviously, } \\
\begin{array}{c}
P \sim Q \Leftrightarrow Q \sim P \Leftrightarrow P \sim Q^{\perp} \\
\Leftrightarrow P^{\perp} \sim Q \Leftrightarrow P^{\perp} \sim Q^{\perp} .
\end{array}
\end{gathered}
$$

Main Theorem: $P \sim Q$ is equivalent to each of the following equalities or inequalities in (3.2) through (3.17). (We shall prove only sufficiency; the proof that $P \sim Q$ implies (3.2) through (3.17) is straightforward and will be omitted).

$$
P \wedge Q=P \sqcap Q \text {. }
$$

Proof: (3.2) is the same as

$$
E_{1}(P Q P)=E_{0} \perp(P Q P),
$$

which means that $P Q P$ is the projection onto $P \wedge Q$. Because of $E_{0}(P Q P)=E_{0}(Q P), P Q P$ and $Q P$ coincide on $(P \wedge Q)^{\perp}$. On the other hand, for every $x \in P \wedge Q, x=Q P x$ and $x=P Q P x$, so that $P Q P$ and $Q P$ are identical.

$$
P \vee Q=P \sqcup Q .
$$

Proof: (3.2) and (3.1).

$$
P=P \wedge Q+P \wedge Q^{\perp} .
$$


Proof: $P \wedge Q^{\perp} \leqq P$ implies $P \wedge Q=P-P \wedge Q^{\perp}$ $=P \wedge\left(P \wedge Q^{\perp}\right)^{\perp}=P \wedge\left(P^{\perp} \vee Q\right)=P \sqcap Q$. Now apply (3.2).

(3.2) is often used to define the 2-place relation $C$ of commensurability

$$
(P, Q) \in C \Leftrightarrow P=P \wedge Q+P \wedge Q^{\perp}
$$

in an orthocomplemented lattice $L_{0}$ (e.g. Mittelstaedt [9], p. 32). It is worth noting that $C$ is symmetrical if and only if $L_{0}$ is quasimodular (some authors say "orthomodular" or "weakly modular"):

$$
P \leqq Q \Rightarrow Q \sqcap P=P .
$$

If this implication holds in $L_{0},(3.1)$ is true in $L_{0}$ (see Mittelstaedt [9], p. 30-34); the reverse implication $Q \sqcap P=P \Rightarrow P \leqq Q$ is always true. If equality of the antecedent is weakened to mere inclusion, we get that $P \sim Q$ is a consequence of

$$
Q \sqcap P \leqq P \text {. }
$$

Proof: $P \wedge Q \leqq Q \sqcap P$ from (2.5) (1); $Q \sqcap P \leqq Q$ together with (3.5) and (3.2) give $P \sim Q$.

$$
P \leqq Q \sqcup P .
$$

Proof: (3.5) and (3.1).

$$
P \leqq(Q \rightarrow P) \text {. }
$$

Proof: $Q \rightarrow P=Q^{\perp} \sqcup P \geqq P$. Apply (3.6) and (3.1). (We remark that always $P \sim(P \rightarrow Q)$; cf. [9], p. 41, 2.40(b)).

$$
P \rightarrow(Q \rightarrow P)=H .
$$

Proof: (3.8) is the same as saying

$$
P^{\perp} \sqcup\left(Q^{\perp} \sqcup P\right)=H .
$$

Because of (2.5) (2) we have also $H=P^{\perp} \vee\left(Q^{\perp} \sqcup P\right)$. But then $P \leqq H$ implies $P \leqq Q^{\perp} \sqcup P$, and (3.6) together with (3.1) yield $P \sim Q$.

$$
P \sqcap Q=Q \sqcap P \text {. }
$$

Proof: Using (3.2), we have to prove that (3.9) implies $P \sqcap Q=P \wedge Q$. We know that always $P \sqcap Q \geqq P \wedge Q$. On the other hand, if $x \in P \sqcap Q$ $=Q \sqcap P$, then $x \in P$ and $x \in Q$ (from the representation (2.3)), i.e. $x \in P \wedge Q$.

$$
P \sqcup Q=Q \sqcup P .
$$

Proof: (3.9) and (3.1).

$$
P \rightarrow Q=Q^{\perp} \rightarrow P^{\perp} .
$$

Proof: (3.11) says $P^{\perp} \sqcup Q=Q \sqcup P^{\perp}$. Apply (3.10) and (3.1).

$$
w_{\varphi}(P \vee Q) \leqq w_{\varphi}(P)+w_{\varphi}(Q)
$$

for all states $\varphi \in H$, where $w_{\varphi}(R):=\langle\varphi, R \varphi\rangle$ for projections $R$ in $H$.

Proof: $P \vee Q=P+\left(P^{\perp} \sqcap Q\right)$ from (2.5) (4), and $w_{\varphi}\left(P^{\perp} \sqcap Q\right) \leqq w_{\varphi}(Q)$ for all states $\varphi$ if and only if $P^{\perp} \sqcap Q \leqq Q$. Using (3.5) and (3.1) gives $P \sim Q$.

(Cf. also Jauch's lemma, p. 117 of [5]).

$$
P=Q \sqcap P+Q^{\perp} \sqcap P .
$$

\section{Proof:}

$$
\begin{aligned}
Q \sqcap P+Q^{\perp} \sqcap P= & \{x \mid P Q x=0\}^{\perp} \\
& +\left\{x \mid P Q^{\perp} x=0\right\}^{\perp} \\
= & \{x \mid P Q x=0 \text { and } \\
& P x-P Q x=0\}^{\perp} \\
= & \{x \mid P x=0 \text { and } \\
& P Q x=0\}^{\perp} \\
= & \left(P^{\perp} \wedge Q^{\perp} \sqcup P^{\perp}\right)^{\perp} \\
= & P \vee(Q \sqcap P) \\
= & P+P^{\perp} \sqcap(Q \sqcap P) .
\end{aligned}
$$

Therefore, (3.13) is equivalent to $P^{\perp} \sqcap(Q \sqcap P)=0$ or $H=P \sqcup\left(Q^{\perp} \sqcup P^{\perp}\right)=P^{\perp} \rightarrow\left(Q \rightarrow P^{\perp}\right)$, which by (3.8) and (3.1) implies $P \sim Q$.

The proof of (3.13) shows also that $P^{\perp} \sqcap(Q \sqcap P)$ $=P^{\perp} \sqcap\left(Q^{\perp} \sqcap P\right)=: I(P, Q)$, which may be called the "interference term", and (3.13) is equivalent to

$$
\begin{aligned}
& I(P, Q)=0 . \\
& P=Q P Q+Q^{\perp} P Q^{\perp} .
\end{aligned}
$$

Proof: Due to (3.13), (3.15) is the same as

$$
Q P Q+Q^{\perp} P Q^{\perp}=Q \sqcap P+Q^{\perp} \sqcap P .
$$

Since always $Q P Q \leqq Q \sqcap P$ and $Q^{\perp} P Q^{\perp} \leqq Q^{\perp} \sqcap P$, the latter equality can only hold if $Q P Q=Q \sqcap P$ and $Q^{\perp} P Q^{\perp}=Q^{\perp} \sqcap P$. But this means that $Q P Q$ is the projection onto $Q \wedge P$, and thus coincides with $P Q$ as was shown in the proof of (3.2).

From $Q P Q+Q^{\perp} P Q^{\perp}=P-\left(Q P Q^{\perp}+Q^{\perp} P Q\right)$ it can be seen that (3.15) holds if and only if $J(P, Q)$ $:=Q P Q^{\perp}+Q^{\perp} P Q$ is zero:

$$
J(P, Q)=0 .
$$

$J(P, Q)$ is the observable which defines Mittelstaedt's probability of interference $([8]$, p. 215):

$$
w_{\varphi}^{\text {int }}(P, Q)=\langle\varphi, J(P, Q) \varphi\rangle,
$$


which is zero if and only if $(J(P, Q)$ is hermitian!) the condition (3.16) holds, i.e. iff $P \sim Q$.

(3.4), (3.13) and (3.15) are saying that each of the following representations for $P$ is equivalent to $P \sim Q$ :

$$
\begin{aligned}
& P=P \wedge Q+P \wedge Q^{\perp}, \\
& P=Q \sqcap P+Q^{\perp} \sqcap P, \\
& P=Q P Q+Q^{\perp} P Q^{\perp} .
\end{aligned}
$$

As our final criterium we show that $P \sim Q$ is equivalent to

$$
P Q P=Q P Q \text {. }
$$

Proof: By (3.2), $P \sim Q$ iff $P \wedge Q=P \sqcap Q$, i.e. $E_{1}(P Q P)=E_{0}(P Q P)$. This equality is true iff $P Q P$ is a projection. But, using (3.17) twice, we get

$$
\begin{aligned}
(P Q P)^{2} & =P Q P P Q P=P Q(P Q P) \\
& =P Q(Q P Q)=P(Q P Q) \\
& =P(P Q P)=P Q P
\end{aligned}
$$

so that the hermitian operator $P Q P$ is idempotent, i.e. in fact a projection.

From the standpoint of physical interpretation, (3.17) is to be expected: $P Q P$ is the defining operator for the "joint" probability of $P$ and (then) $Q$ and determines the conditional probability of $Q$, given $P$. Considering this interpretation, and (3.16), for instance, which is equivalent to (3.17), it comes as no surprise that $P Q P=Q P Q$ should imply $P \sim Q$. Mathematically speaking, however, this implication seems curious: (3.17) means that for $P Q=Q P$ it is sufficient that $P Q$ has the same value for $P x$ as $Q P$ has for $Q x$, for all $x \in H$. In other words, (3.17) permits an implication from the equality of positive self-adjoint operators $P Q P$ and $Q P Q$ to the equality of prima facie more general operators $P Q$ and $Q P$. Putting $A=P Q, A^{*}=Q P,(3.17)$ may be restated as: $A=A^{*}$ is equivalent to $A A^{*}=A^{*} A$, i.e. for $A=P Q$ self-adjointness and normality are the same.

For this reason it may be of interest to have a proof of (3.17) independent of (3.2) and of the representation of $P \sqcap Q$ and $P \wedge Q$ through the spectral measure of $P Q P$. We shall do so now.

Proposition. For any two projections $P$ and $Q$ in a complex Hilbert space $H$, the commutativity relation $P Q=Q P$ is equivalent to $P Q P=Q P Q$.
Again, we need only prove the non-trivial direction.

We need the following

Lemma: Let $A$ and $B$ be bounded linear operators in $H$ such that

(1) $A B=B A$,

(2) $A^{2}=B^{2}$, and

(3) $(A-B)=-(A-B)^{*}$.

Then (4) $E$ commutes with any transformation that commutes with $A-B$, and (5) $A=(2 E-I) B$, where $E$ is the orthogonal projection onto the nullspace $M=E_{0}(A-B)$ of $A-B$.

Proof of the Lemma: (Cf. [1], p. 424, Theorem 23.3 ; note that in our proof $A$ and $B$ need not be self-adjoint!). Suppose that $C$ commutes with $A-B$. This implies $C(M) \subset M$. From

$$
\begin{aligned}
C(A-B) & =(A-B) C \Rightarrow(A-B)^{*} C^{*} \\
& =C^{*}(A-B)^{*}
\end{aligned}
$$

and (3), we have

$$
(A-B) C^{*}=C^{*}(A-B),
$$

which implies $C^{*}(M) \subset M$. Therefore $C$ reduces $M$, i.e. $C E=E C$, proving (4).

From (1) and (2) we have

$$
(A-B)(A+B)=A^{2}-B^{2}=0,
$$

i.e. (6)

$$
E(A+B)=A+B .
$$

For any vector $z \in H$ write $z=x+y$, where $x \in M$ and $y \in M^{\perp}$. It follows

$$
E(A-B) z=E(A-B) x+E(A-B) y .
$$

The first term on the right is zero, because $x \in M$ $=E_{0}(A-B)$ and the second is zero because $E$ commutes with $A-B$, according to (4).

Hence

(7) $\quad E(A-B)=0$.

Combining (6) and (7), gives

$$
E(A+B)-E(A-B)=A+B
$$

or

$$
A=2 \cdot E B-B=(2 E-I) B,
$$

which proves (5). 
We wish to apply the Lemma for $A=P Q$, $B=Q P$. Assumption (1) is the same as $P Q P$ $=Q P Q$. Using this, and observing

$$
\begin{aligned}
(P Q)^{2} & =P Q P Q=P P Q P=P Q P \\
& =Q P Q=(Q P)^{2},
\end{aligned}
$$

we note that $(2)$ is fulfilled.

Moreover,

$$
\begin{aligned}
(P Q-Q P)^{*} & =Q P-P Q \\
& =-(P Q-Q P),
\end{aligned}
$$

which is assumption (3) of our Lemma.

Proof of the Proposition: From

$$
\begin{aligned}
(P Q P)^{2} & =P Q P P Q P=P Q P Q P \\
& =P Q Q P Q=(P Q)^{2}
\end{aligned}
$$

and (8), we see that $P Q P=Q P Q$ must be a projection. We claim that $P Q P$ is the projection onto $P \wedge Q$. This may be seen from

$$
P Q P=(P Q P)^{2}=(P Q)^{2},
$$

i.e.

$$
P Q P=(P Q)^{2 k}, \quad k \geqq 1,
$$

and from the fact that the projection onto $P \wedge Q$ is given by the limit of $(P Q)^{n}, n \rightarrow \infty$.

We prove $P Q=Q P$.

If $z \in P \wedge Q$, trivially $P Q z-Q P z=z-z=0$. If $z \in P^{\perp} \vee Q^{\perp}$, write $z=\lim \left(x_{n}+y_{n}\right)$, where $x_{n} \in P^{\perp}$ and $y_{n} \in Q^{\perp}$. Using (5) of our Lemma gives

$$
\begin{aligned}
& P Q=(2 E-I) Q P \text { and } \\
& Q P=(2 E-I) P Q,
\end{aligned}
$$

[1] G. Bachmann and L. Narici, Functional Analysis, Academic Press, London 1966.

[2] E. G. Beltrametti and G. Cassinelli, On state transformations induced by yes-no experiments, in the context of quantum logic, J. Phil. Logic 6, 369 (1977).

[3] J. Bub, Von Neumann's Projection Postulate as a Probability Conditionalization Rule in Quantum Mechanics, J. Phil. Logic 6, 381 (1977).

[4] M. Jammer, The Philosophy of Quantum Mechanics, John Wiley, New York 1974.

[5] J. M. Jauch, Foundation of Quantum Mechanics, Addison Wesley, Reading, Mass., 1968.

[6] H. Kröger, Zwerch-Assoziativität und verbandsähnliche Algebren, S.B. Bayer. Akad. Wiss., München 1973. where $E$ is the orthogonal projection onto

$$
E_{0}(P Q-Q P)=E_{0}(Q P-P Q)
$$

(note that the assumptions of the Lemma are sym. metrical in $A, B$ ).

Therefore, using continuity,

$$
\begin{aligned}
P Q z & =\lim _{\mathrm{n}}\left(P Q x_{n}+P Q y_{n}\right) \\
& =\lim _{\mathrm{n}} P Q x_{n}=\lim _{\mathrm{n}}(2 E-I) Q P x_{n}=0,
\end{aligned}
$$

similarly $Q P x=0$

Hence, $P Q$ and $Q P$ coincide on $H$.

\section{Remark}

It should be noted that the Proposition is also a special case of a rather deep theorem by FugledePutnam, Rosenblum (cf. [11], p. 300, Theorem 12.16, where Rosenblum's proof is given):

Assume that $A, B, T$ are bounded transformations on $H, A$ and $B$ are normal, and

$$
A T=T B \text {. }
$$

Then $A^{*} T=T B^{*}$.

Taking $A=P Q, \quad B=Q P, \quad T=P$ yields our Proposition.

\section{Acknowledgement}

I am indebted to Dr. E.-W. Stachow, of Cologne University, who kindly pointed out to me the two references [6] and [7] where the conditional connectives have been studied in great detail, and in a more general setting than Hilbert space.

[7] H. Kröger, Das Assoziativgesetz als Kommutativitätsaxiom in Boole'schen Zwerchverbänden, J. Reine Angew. Math. 285, 53 (1974)

[8] P. Mittelstaedt, Philosophische Probleme der modernen Physik, BI Mannheim, 1976. Engl. Transl. as Philosophical Problems of Modern Physics, Reidel, Dordrecht 1976.

[9] P. Mittelstaedt, Quantum Logic, Reidel, Dordrecht 1978.

[10] C. Piron, Foundations of Quantum Physics, Reading, Mass., 1976.

[11] W. Rudin, Functional Analysis, McGraw-Hill, New York 1973. 